\title{
First products of DNA marker-assisted selection in sorghum released for cultivation by farmers in sub-saharan Africa
}

\author{
Abdalla Mohamed ${ }^{1 *}$, Rasha Ali ${ }^{2}$, Osama Elhassan², Elnayer Suliman², Charles Mugoya ${ }^{3}$, Clet W. Masiga ${ }^{3}$, Adil Elhusien ${ }^{4}$ and \\ Charles T. Hash ${ }^{5}$ \\ *Correspondence: A.H.Moahmed@cgiar.org

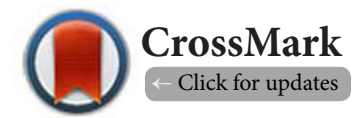 \\ 'International Crops Research Institute for the Semi-Arid Tropics (ICRISAT), Addis Ababa-Ethiopia. \\ ${ }^{2}$ Agricultural Research Corporation (ARC), Wad Medani, Sudan. \\ ${ }^{3}$ Agro-biodiversity and Biotechnology Program (AGROBIO), Association for Strengthening Agricultural Research in East and Central \\ Africa (ASARECA), Entebbe, Uganda. \\ ${ }^{4}$ Department of Botany, Faculty of Science, University of Khartoum, Khartoum, Sudan. \\ ${ }^{5}$ International Crops Research Institute for the Semi-Arid Tropics (ICRISAT), Patancheru, Andhra Pradesh, India.
}

\begin{abstract}
Striga hermonthica (Del.) Benth. is the major biotic constraint to sorghum production. Its control is difficult and can only be achieved through integrated management strategies that depend mainly on host plant resistance and enhanced soil fertility. However, breeding for resistance is hampered by the complexity of host parasite interactions and lack of reliable screening methods. The invention of molecular markers has enhanced the effectiveness of breeding for resistance. Five genomic regions (QTLs) with linked markers associated with Striga resistance were mapped in sorghum variety N13 by [10]. In this study, to increase the efficiency of marker-assisted selection (MAS), 27 EST-SSR markers in close association with Striga resistance QTLs were also identified and mapped. Populations of backcross $\left(\mathrm{BC}_{3} \mathrm{~S}_{4}\right)$ derived from N13 (Striga resistant) X three farmer preferred sorghum cultivars: Tabat, Wad Ahmed and AG-8 (Striga susceptible) were generated. Thirty-one lines $\left(\mathrm{BC}_{3} \mathrm{~S}_{4}\right)$ with confirmed Striga field resistance were genotyped with foreground and background selection makers. Twenty resistant lines, with two or more major QTLs were selected for regional evaluation. Of these 10 lines were selected and advanced for multi-location testing, together with Wad Ahmed, Tabat, AG-8, N13, SRN39 and IS9830 as checks. Standard variety trials were conducted in Striga sick plots over three seasons (2009-2011) in Sudan, Gezira Research Station, Damazine, Sinnar, and Gedarif. Results revealed that four lines $\left(\mathrm{T}_{1} \mathrm{BC}_{3} \mathrm{~S}_{4}, \mathrm{AG}_{6 \mathrm{BC}} \mathrm{S}_{4}, \mathrm{AG}_{2} \mathrm{BC}_{3} \mathrm{~S}_{4}\right.$ and $\mathrm{W}_{2} \mathrm{BC}_{3} \mathrm{~S}_{4}$ ) were Striga resistant and agronomically superior with yields ranging from $180 \%$ to $298 \%$ higher relative to their recurrent parents. This Striga resistance coupled with superior attributes of the recurrent parent (including very high yield potentials, high grain quality and drought tolerance) will provide adaptation and stability across a wide range of environments. These are the first products of DNA markerassisted selection (MAS) in sorghum released for cultivation by farmers in sub-Saharan Africa.
\end{abstract}

Keywords: Striga hermonthica, molecular markers, quantitative trait loci (QTL), simple sequence repeat (SSR)

\section{Introduction}

Sorghum (Sorghum bicolor (L.) Moench) is a drought tolerant crop that evolved various ecotypes that withstand an array of biotic and a biotic stresses $[16,17]$. Drought tolerance and the ability to withstand harsh environments enable sorghum to grow under severe stress conditions. The relative adaptation to harsh environments makes sorghum a crop of outstanding potential to meet the increasing demand of food globally. However, sorghum yield is reduced considerably by both biotic and abiotic stresses $[16,17]$. Due to its adaptation to marginal, hot, and drought prone areas, sorghum consumption is highest in the poorest and the most food-insecure regions of the world [6]. Moreover, recent climatic changes make sorghum a major hope to meet the challenge of anticipated food shortage.

Witch weed (Striga sp.), is a serious parasitic angiosperm of many cereal crops. It is the most limiting biotic factor in the production of sorghum in semi-arid regions of Sub-Saharan Africa. Parasitic weeds damage $70-100 \%$ of staple food crops in 
Mohamed et al. Journal of Plant Science \& Molecular Breeding 2014,

the semi-arid tropics of Africa and Asia [5]. In Sudan, the losses in yield of cereal grain crops due to $S$. hermonthica may reach up to $100 \%$ in heavily infested soils [7]. Striga is an intractable problem because of the complex host-parasite interactions, production of large number of seeds with prolonged viability, and special germination and development requirements [18]. A number of control measures that have been tried are either not successful or are not feasible economically. Integrated management strategies with host plant resistance as their backbone are believed to be the only solution $[5,8]$. However, this integrated approach had limited success, since efforts to identify germplasm with resistance to Striga parasitism generally failed. This is due to the difficulty in selection for resistance in field tests, where unpredictable environmental factors influence Striga infestation. Nevertheless, a few Striga resistant varieties with widely effective field resistance were identified; these include SRN39, IS9830, Framida, 555 and N13. The first four of these five genotypes possess low germination stimulant production as a mechanism of resistance, and the last provides mechanical barrier. N13 is a unique source for resistance against Striga, because it possesses post-germination Striga resistance mechanism(s) that affects Striga seed reserves in the soil [15]. However, these resistant varieties are generally low yielders and lack adaptation to Striga-infested areas [5].

The recent availability of molecular markers made it possible to breed for Striga resistance with precision in selection [5]. In the past, molecular markers have been used in sorghum to identify quantitative trait loci (QTL) for many complex traits such as Striga resistance. However, progress in utilizing molecular markers associated with these QTLs has been limited due to the lack of saturated genetic map for the various linkage groups of sorghum. Fortunately, the genetic map of sorghum has recently become standardized and fairly saturated with molecular markers linked to the specific chromosomal regions [19]. The approach of QTL mapping is advantageous as genomic regions affecting complex quantitative traits such as Striga resistance, can be identified and used for the development of improved sorghum cultivars through marker-assisted selection (MAS).

In the past, five QTLs underlying different Striga resistant phenotypes and molecular SSR markers associated with specific region have been identified and assigned to their specific chromosomal location [10]. These regions and associated SSR markers have been used in advanced backcross populations allowing exploitation of diverse resistance sources [20]. Improving precision of selection and marker assisted backcross breeding would be particularly handy because the procedure considerably shortens the time required to introgress genes into elite cultivars. The present study was aimed at utilizing modern biotechnology tools to identify, map and locate QTLs for Striga resistance which can be exploited through MAS to breed Striga resistant sorghum varieties. The specific objectives were to develop and map SSR and DArT markers tightly linked to previously identified Striga resistance QTLs and to develop and evaluate Striga resistant sorghum lines for commercial release.

\section{Materials and methods Germplasm}

Three populations of backcross-derived lines $\left(\mathrm{BC}_{3} \mathrm{~S}_{4}\right)$ from crosses of $\mathrm{N} 13$ (Striga resistant) with farmer preferred sorghum cultivars i.e., Tabat, Wad Ahmed and AG-8 (Striga susceptible) were developed using backcrossing (Figure 1). These lines $\left(\mathrm{BC}_{3} \mathrm{~S}_{4}\right)$ along with the parents were phenotyped in Striga sick plots. $\left(\mathrm{BC}_{3} \mathrm{~S}_{4}\right)$ Striga resistant lines (31) were genotyped with 89 markers (EST-derived SSR markers and DArT markers) linked with putative Striga resistance QTLs.

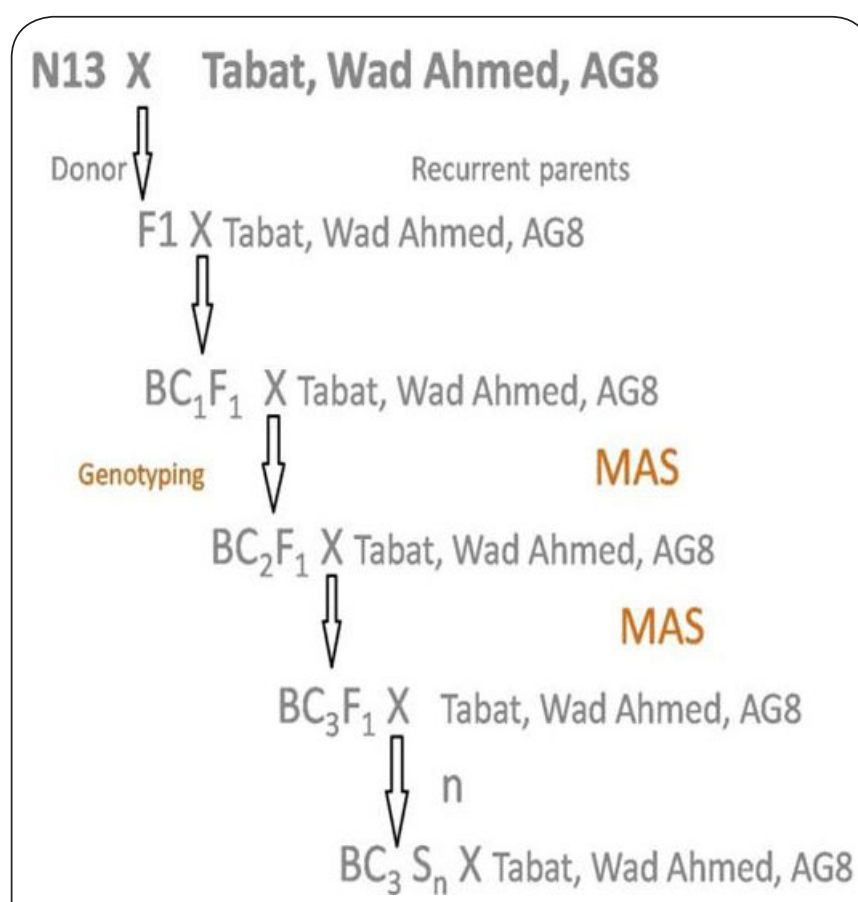

Figure 1. Crossing scheme used to generate $\mathrm{BC}_{3} \mathrm{~S}_{4}$ populations.

\section{Molecular markers analysis \\ DNA extraction}

A high-throughput mini-DNA extraction protocol was followed for extraction DNA from parental lines and $\mathrm{BC}_{3} \mathrm{~S}_{4}$ populations using modified CTAB method as described [14].

\section{Genotyping}

Microsatellites: SSR marker amplifications were performed in $5 \mu \mathrm{l}$ reaction volumes consisting of $1 \mu \mathrm{l}$ of $5 \mathrm{ng}$ DNA template, $0.25 \mu \mathrm{l}$ of $2 \mathrm{mM}$ dNTPs, $0.2 \mu \mathrm{l}$ OF $2 \mathrm{pm} / \mu \mathrm{l} \mathrm{M} 13$ tailed forward primer: $0.4 \mu \mathrm{l}$ OF $2 \mathrm{pm} / \mu \mathrm{l} \mathrm{M} 13$ tailed reverse primer: $0.4 \mu \mathrm{l} \mathrm{OF}$ $2 \mathrm{pm} / \mu \mathrm{l} \mathrm{M} 13$ labe, $0.1 \mathrm{U}(0.2 \mu \mathrm{l}$ of $5 \mathrm{U} / \mu \mathrm{l})$ of Taq DNA polymerase (Sib-Enzymes, Russia), $0.5 \mu \mathrm{l}$ of 10X PCR buffer (Sib Enzymes, Russia), $0.25 \mu \mathrm{l}$ of $50 \mathrm{mM} \mathrm{MgCl}$ (Sib Enzymes, Russia). In 
addition, fluorescent dye phosphoramidite, either 6-FAM (blue), VIC (green), NED (yellow), PET (red) were used in the $P C R$ reaction mixture for detection of the amplified product on $A B I 3700 / 3130$ analyzer. The cycling conditions for PCR on a Gene Amp ${ }^{\otimes}$ PCR System 9700 (PE-Applied Biosystems) thermal cycler were optimized to initial denaturation of 15 min at $94^{\circ} \mathrm{C}$, followed by 10 cycles (touchdown) of $94^{\circ} \mathrm{C}$ for $15 \mathrm{sec}$, annealing touchdown temperature reducing from 61 to $51^{\circ} \mathrm{C}$ for $20 \mathrm{sec}$ over $10 \mathrm{cycles}$, with extension at $72^{\circ} \mathrm{C}$ for $30 \mathrm{sec}$. This was followed by denaturation at $94^{\circ} \mathrm{C}$ for $10 \mathrm{sec}$, annealing at $54^{\circ} \mathrm{C}$ for $20 \mathrm{sec}$, and extension at $72^{\circ} \mathrm{C}$ for $30 \mathrm{sec}$ for 34 cycles, followed by final extension of $20 \mathrm{~min}$ at $72^{\circ} \mathrm{C}$. As described by $[11,12]$, the amplified primers were visualized on Agarose and detected on ABI 3730 analyzer (Figures 2a and 2b).

\section{Field evaluation}

Based on Striga resistance and their high yield potential, lines $\mathrm{T} 1 \mathrm{BC}_{3} \mathrm{~S}_{4^{\prime}} \mathrm{AG}_{2} \mathrm{BC}_{3} \mathrm{~S}_{4^{\prime}}, \mathrm{AG} \mathrm{BC} \mathrm{C}_{3} \mathrm{~S}_{4}$ and $\mathrm{W} 2 \mathrm{BC}_{3} \mathrm{~S}_{4}$ Advanced sorghum variety trials included Wad Ahmed, Tabat, AG-8, N13, SRN39 (Muggaem Buda1) and IS9830 (Muggaem Buda2) as checks. The trials were conducted under nine environments, i.e., Wad-Madani, Damazine, Sinnar and Gadarif. The lines T1BC3S5, $\mathrm{AG} 2 \mathrm{BC} 3 \mathrm{~S} 5, \mathrm{AG} 6 \mathrm{BC} 3 \mathrm{~S} 5$ and $\mathrm{W} 2 \mathrm{BC} 3 \mathrm{~S} 5$, were also tested together with Ajab Sedo and Korokolo as checks, in verification yield trials in 2 locations at Gadarif in season 2010/2011. The standard cultural practices for sorghum at Gezira Research Farm were followed. Land was prepared by disc ploughing, disc harrowing, leveling and ridging in irrigated sites and by disc harrowing in rain-fed sites. Treatments were laid in randomized complete block design with three replicates. Planting was made during the first two weeks of July on ridges in irrigated sites and on flat in rain-fed sites, at spacing of $80 \mathrm{~cm}$ between rows and $30 \mathrm{~cm}$ between plants at 3 plants/hole (population density of 125000 plants/ha). In irrigated trials, $40 \mathrm{~kg}$ urea/ha was applied. For artificial infestation, Striga seeds were mixed with soil at 1 $\mathrm{mg} / \mathrm{kg}$, and the mixture was planted at $5 \mathrm{~g} / \mathrm{hole}$. The crop was kept weed-free and irrigated every two weeks or whenever necessary. Irrigation was stopped three weeks before harvest. Assessments were made in the central rows of each plot. Data included; Striga count, days to $50 \%$ bloom and plant height for the trials. At harvest, heads were cut, sun-dried, threshed, weighed, and 1000 grain weight was determined.

General Linear Method (GLM) was used for statistical analysis for all experiments unless it is noted otherwise.

\section{Assessment of grain quality}

Grain samples were subjected to physical analysis according to the American Association of Cereal Chemists [1]. Extraction rate, as a result of decortication/dehulling of grains for two minutes using a Tangential Abrasive Dehulling Device (TADD), was used as measure grain hardness [4]. Sorghum samples for chemical composition were milled into whole meal flour, using KT type 120 mills. Moisture and ash contents of whole meal were determined according to the [1]. Protein content was determined by semi-micro kjeldahl according to standard methods of the [2]. Carbohydrates, total acidity and tannins content were determined according to standard methods of the [2].

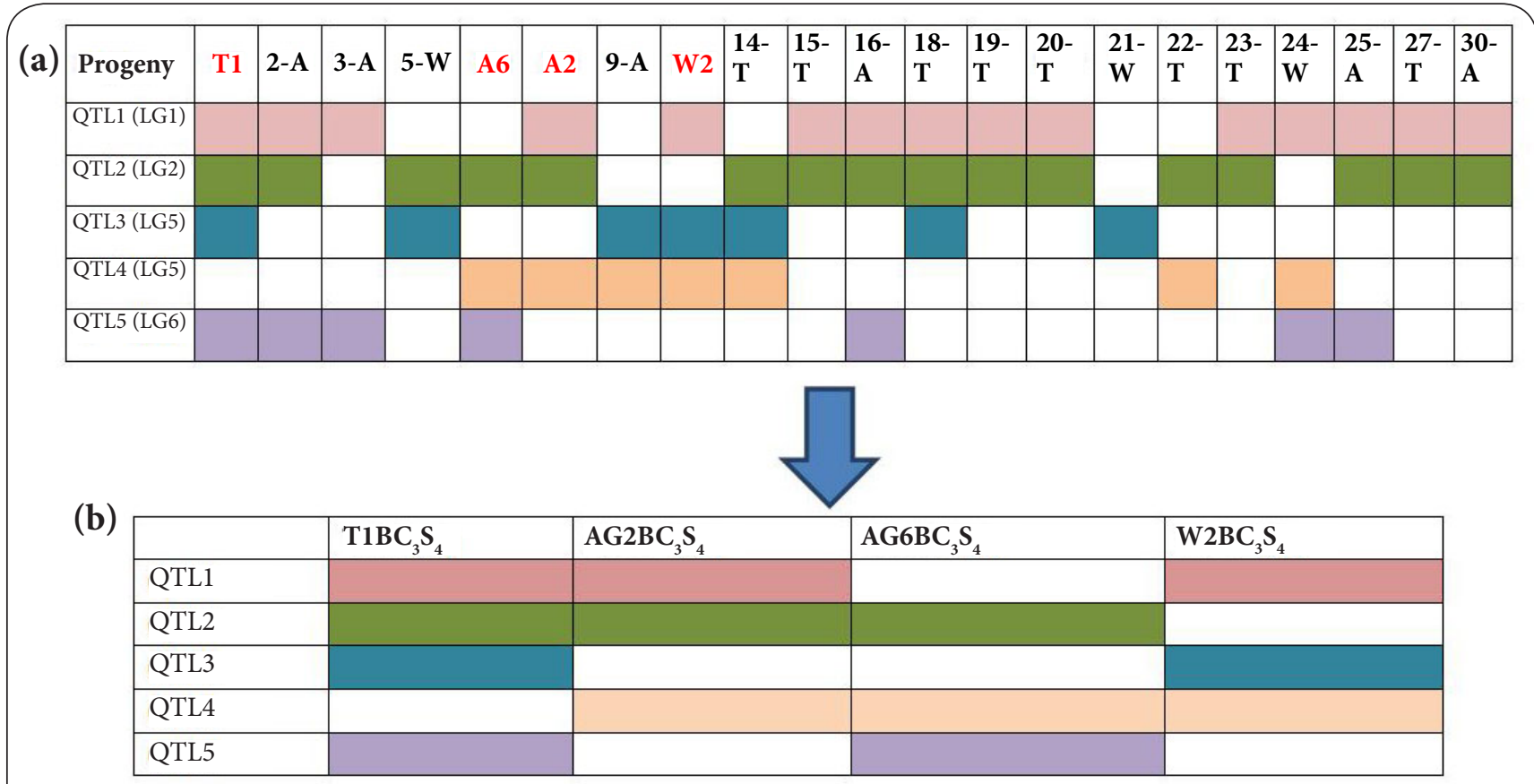

Figure 2. Foreground and background selection using SSR markers. (a). $\left(20 \mathrm{BC}_{3} \mathrm{~S}_{4}\right),(\mathbf{b}) .\left(4 \mathrm{BC}_{3} \mathrm{~S}_{4}\right)$. 
Mohamed et al. Journal of Plant Science \& Molecular Breeding 2014,

\section{Results and dicussions Fine mapping}

In this era of molecular biology Striga resistance was introgressed from an exotic donor parent N13 into farmer preferred sorghum variety backgrounds using marker-assisted selection (MAS) and backcrossing. Accordingly, 186 SSR markers have been identified and show polymorphism across different sorghum backgrounds. Out of these, 17 markers in close association with Striga resistance QTLs have been mapped. Also, 139 DArT markers associated with Striga resistant QTLs have been identified.

Polymorphic EST-SSR markers (186) have been identified across 182 Recombinant Inbred Lines (RILs) from the cross N13 x E36-1. Out of these, 27 EST-SSR markers in close association with Striga resistance QTLs were mapped. $\mathrm{BC}_{3} \mathrm{~S}_{4}$ lines (31) along their parents, (N13, Tabat, Wad Ahmed and AG-8) were screened with 186 EST-SSR and 420 DArT markers. This achievement has been used to increase the efficiency of marker assisted selection (MAS) by saturating the genetic linkage map at Striga resistance regions (QTLs). These efforts provided useful information for improving precision of MAS, simultaneously, tagged Striga resistance QTLs were introgressed in Backcrossing is the fastest way to recover the genome of the recurrent agronomically favorable parent.

$\mathrm{BC}_{3} \mathrm{~S}_{4}$ generated lines (31) from the backcrossing scheme with confirmed Striga field resistance were genotyped with markers for selecting homozygous progenies for the donor parent alleles at QTL regions (foreground selection) and markers to select homozygous progenies for the recurrent parent alleles in much of the non-QTL regions (background selection) (Figures 2a, 2b and 3). Superior $\mathrm{BC}_{3} \mathrm{~S}_{4}$ lines (31) with two or more Striga resistance QTLs were first selected. Striga resistant and agronomically superior genotypes (10) were selected and advanced together with Wad-Ahmed, Tabat, AG-8, N13, SRN39 and IS9830 as checks for multi-location trials in nine environments, under natural or artificial Striga infestation). Results revealed that sorghum lines $\mathrm{T}_{1} \mathrm{BC}_{3} \mathrm{~S}_{4^{\prime}}$ $\mathrm{AG} 2 \mathrm{BC}_{3} \mathrm{~S}_{4}, \mathrm{AG}_{6} \mathrm{BC}_{3} \mathrm{~S}_{4}$ and $\mathrm{W} 2 \mathrm{BC}_{3} \mathrm{~S}_{4}$ were Striga resistant and with high grain yield potential (Figure 3 ).

\section{Grain yield}

Analysis of variance of individual environments revealed significant differences $(P=0.05)$ among lines and checks in the five irrigated, and four rain-fed environments. Mean grain yield for each genotype at each environment is presented in

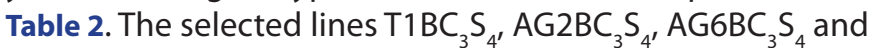
$\mathrm{W} 2 \mathrm{BC}_{3} \mathrm{~S}_{4}$ consistently produced higher grain yields in all Striga infested areas, and their yield exceeded the checks (Tables 2 and 3$)$. The highest grain yield $(14720.3 \mathrm{~kg} / \mathrm{h})$ was produced by $\mathrm{WBC}_{3} \mathrm{~S}_{4^{\prime}}$ at Sinnar in season 2010, while the lowest yield $(69.805 \mathrm{~kg} / \mathrm{h})$ was attained by Tabat at GRS Wad-Madani in season 2009. Grain yield in all environments indicated superiority of the 4 lines over the checks (Table 3 ). For the nine environments entries ranked as follows: $\mathrm{W}_{2} \mathrm{BC}_{3} \mathrm{~S}_{4}>\mathrm{T} 1 \mathrm{BC}_{3} \mathrm{~S}_{4}>$

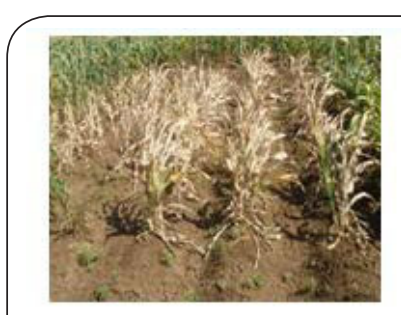

Tabat

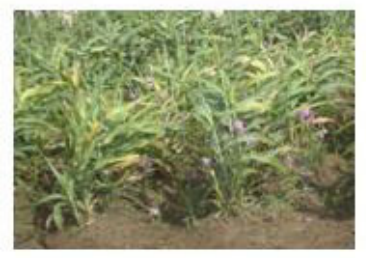

Wad Ahmed

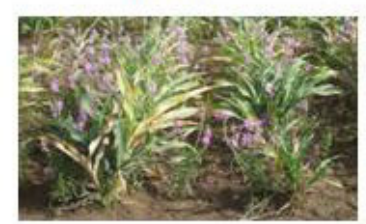

AG8

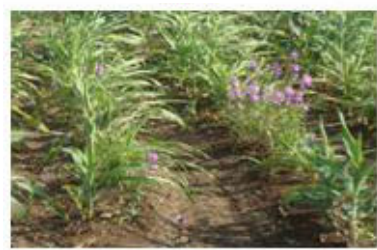

AGB

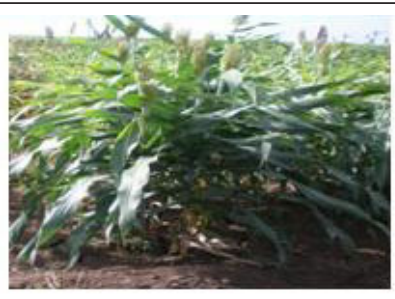

$\mathrm{T}_{1 B C} \mathrm{~S}_{4}$

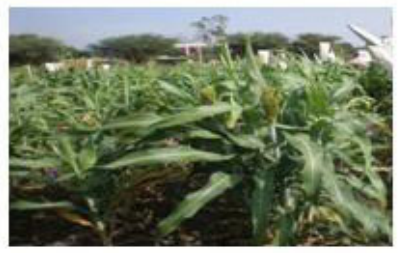

$\mathrm{W}_{2} \mathrm{BC}_{3} \mathrm{~S}_{4}$

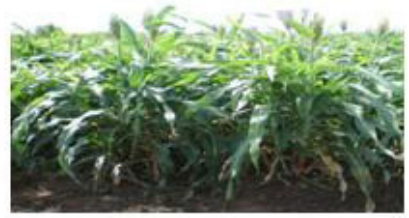

AG2BC3

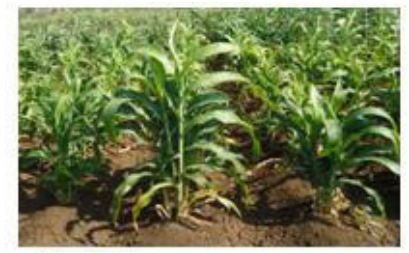

AG6 $\mathrm{BC}_{3} \mathrm{~S}_{4}$
Figure 3. Phenotypic evaluation of $\mathrm{BC}_{3} \mathrm{~S}_{4}$ lines with the parents, in artificially Striga infested plot.

Table 1. Rainfall (mm) during the testing period.

\begin{tabular}{llllllll}
\hline Month & \multicolumn{2}{c}{2009} & \multicolumn{3}{c}{ 2011 } & \multicolumn{2}{c}{2010} \\
\hline & DAM & SGAD & NGAD & DAM & SGAD & NGAD & DAM \\
\hline May & 27 & -- & 8.9 & 125 & 0 & 0 & 0 \\
June & 125 & 77 & 50.2 & 180 & 0 & 0 & 140 \\
July & 129 & 261 & 191.6 & 40 & 92 & 63.5 & 102.6 \\
August & 222 & 241 & 276 & 81 & 176.6 & 101.4 & 189 \\
September & 79 & 181 & 84.9 & 180 & 25.7 & 98.8 & 114 \\
October & 62 & 17 & 17.6 & 59 & 0 & 20.8 & 29 \\
Total & 643 & 777 & 627.8 & 665 & 294.3 & 284.5 & 575.6 \\
\hline
\end{tabular}

DAM=Damazin; NGAD=North Gadarif; SGAD=South Gadarif

AG2BC3S4>the checks (Table 3). It is obvious that these lines are not only Striga resistant but also unlock the productivity potentials of their recurrent parents in the Striga endemic areas. Results of combined analysis of variance for grain yield across the nine environments are presented in Table 4. The mean squares of genotypes, environments and genotypes $x$ environments interactions are highly significant $(P=0.01)$. 
Mohamed et al. Journal of Plant Science \& Molecular Breeding 2014, http://www.hoajonline.com/journals/pdf/2050-2389-3-3.pdf

Table 2. Mean grain yield $(\mathrm{kg} / \mathrm{h})$ of entries, averaged over nine environments.

\begin{tabular}{|c|c|c|c|c|c|c|c|c|c|c|}
\hline & & & & & & & & DAM011 & GAD011 & COMB \\
\hline $\mathrm{T}_{1 \mathrm{BC}_{3} \mathrm{~S}_{4}}$ & & & & & & & $970.25^{a}$ & & $04.16^{\mathrm{a}}$ & $2465.44^{\mathrm{bc}}$ \\
\hline $\mathrm{AG}_{1 B C} \mathrm{~S}_{4}$ & $10607 c$ & $272.90^{\mathrm{b}}$ & $2278.45^{\mathrm{ed}}$ & $3401.73^{\text {bcde }}$ & $3470.754^{\text {bcde }}$ & $1166.20^{\mathrm{abc}}$ & $446.65^{\mathrm{de}}$ & $668.78^{\mathrm{b}}$ & $616.42^{\mathrm{a}}$ & $1858.78^{\mathrm{f}}$ \\
\hline $\mathrm{AG} 2 \mathrm{BC}_{3} \mathrm{~S}_{4}$ & $597.36^{c}$ & $350.65^{\mathrm{b}}$ & $2439.5^{\text {cde }}$ & $4291.14^{\mathrm{ab}}$ & $4318.272^{\mathrm{ab}}$ & 1477 9 ab & $760.01^{\mathrm{abc}}$ & $1685.04^{\mathrm{a}}$ & $1131.93^{\mathrm{a}}$ & $2338.83^{\mathrm{cd}}$ \\
\hline & & $397.85^{\mathrm{a}}$ & & & & & & & & \\
\hline W1I & $1013.67^{c}$ & & $2222.13^{\mathrm{de}}$ & & $3262.504^{\mathrm{de}}$ & & $426.02^{\mathrm{de}}$ & & & \\
\hline AG4B & & $2398.25^{\mathrm{b}}$ & $2322.88^{\text {cde }}$ & $3401.73^{\text {cde }}$ & $3679.004^{\text {cde }}$ & $836.97^{\mathrm{abc}}$ & $413.33^{\mathrm{de}}$ & $909.95^{\mathrm{b}}$ & & $1893.77^{\mathrm{f}}$ \\
\hline $\mathrm{AG}_{5} \mathrm{BC}_{3} \mathrm{~S}_{4}$ & $1007.00_{c}$ & $2293.53^{\mathrm{b}}$ & $2263.38^{\mathrm{de}}$ & $3722.08^{\mathrm{bcd}}$ & $3818.234^{\mathrm{bcd}}$ & $1531.93^{\mathrm{ab}}$ & $395.08^{\mathrm{de}}$ & $1197.93^{\mathrm{ab}}$ & $1237.12^{\mathrm{a}}$ & $2095.11^{\mathrm{e}}$ \\
\hline $\mathrm{AG} \mathrm{BC}_{3} \mathrm{~S}_{4}$ & $1469.00^{\mathrm{b}}$ & $3172.54^{\mathrm{a}}$ & $2833.79^{b c}$ & $4372.77^{\mathrm{ab}}$ & $4442.746^{\mathrm{ab}}$ & $1523.99^{\mathrm{ab}}$ & $674.33^{\mathrm{bcd}}$ & $779.85^{\mathrm{b}}$ & $1799.99^{\mathrm{a}}$ & $2566.35^{\mathrm{b}}$ \\
\hline $\mathrm{T} 2 \mathrm{~B}$ & 1046. & $172.15^{\mathrm{b}}$ & $2157.07^{\mathrm{e}}$ & $3104.71^{\mathrm{de}}$ & $3262.504^{\mathrm{de}}$ & & & $771.12^{\mathrm{b}}$ & $1226.18^{\mathrm{a}}$ & $1866.87^{\mathrm{f}}$ \\
\hline W21 & 1651 & & & $4720.25^{\mathrm{a}}$ & $4650.996^{a}$ & & & $1175.72^{\mathrm{ab}}$ & $1714.08^{\mathrm{a}}$ & $2820.30^{\mathrm{a}}$ \\
\hline SRI & & & & & & & & & & \\
\hline IS9830 & & & & & & & & & & \\
\hline N13 & & & & $2826.25^{\mathrm{de}}$ & & & & & & $1507.97^{\mathrm{gh}}$ \\
\hline Tabat & & & & & & & & & & \\
\hline W. Ahmed & & & & & & $1079.57^{\mathrm{abc}}$ & & & $1284.01^{\mathrm{a}}$ & $1477.03^{\mathrm{h}}$ \\
\hline & & & & & & & & $716.38^{\mathrm{b}}$ & $1233.32^{\mathrm{a}}$ & $1428.71^{\mathrm{h}}$ \\
\hline Mean & 952.75 & 060.84 & 1987.54 & 3405.02 & 3470.2542 & 1241.22 & 547.95 & 964.19 & 1133.38 & 1896.84 \\
\hline SE \pm & 37.02 & 113.74 & 115.74 & 208.73 & 226.9568 & 147.73 & 58.81 & 131.26 & 288.93 & 168.27 \\
\hline
\end{tabular}

** Means with different letters in the same column are significantly different at $P=0.05$

$\mathrm{MED}=$ Madani; $\mathrm{SIN}=$ Sinnar; $\mathrm{DAM}=$ Damazine; $\mathrm{GAD}=$ Gadarif

Table 3. Level of superiority of sorghum genotypes for grain yield in across environments.

\begin{tabular}{lccccccccccc}
\hline ENT/OB & A & ab & abc & B & bc & bcd & c & cd/d & e/ed & ef/f/g/h & Ranking \\
\hline W2BC $_{3} \mathrm{~S}_{4}$ & 8 & 1 & 0 & 0 & 0 & 0 & 0 & 0 & 0 & 0 & 1 \\
T1BC $_{3} \mathrm{~S}_{4}$ & 3 & 2 & 2 & 1 & 1 & 0 & 0 & 0 & 0 & 0 & 2 \\
AG6BC $_{3} \mathrm{~S}_{4}$ & 2 & 3 & 0 & 2 & 2 & 0 & 0 & 0 & 0 & 0 & 3 \\
AG2BC $_{3} \mathrm{~S}_{4}$ & 2 & 2 & 2 & 1 & 0 & 0 & 1 & 0 & 1 & 0 & 4 \\
AG3BC $_{3} \mathrm{~S}_{4}$ & 3 & 2 & 1 & 0 & 0 & 0 & 0 & 0 & 3 & 0 & 5 \\
AG5BC $_{3} \mathrm{~S}_{4}$ & 1 & 1 & 1 & 1 & 0 & 2 & 1 & 0 & 2 & 0 & 6 \\
AG1BC $_{3} \mathrm{~S}_{4}$ & 1 & 0 & 1 & 2 & 0 & 2 & 1 & 0 & 2 & 0 & 7 \\
W1BC $_{3} \mathrm{~S}_{4}$ & 1 & 0 & 1 & 2 & 0 & 0 & 1 & 0 & 4 & 0 & 8 \\
AG4BC $_{3} \mathrm{~S}_{4}$ & 1 & 0 & 1 & 2 & 0 & 1 & 1 & 0 & 3 & 0 & 9 \\
T2BC $_{3} \mathrm{~S}_{4}$ & 1 & 0 & 1 & 2 & 0 & 0 & 1 & 0 & 4 & 0 & 10 \\
SRN39 & 1 & 0 & 1 & 1 & 1 & 0 & 0 & 0 & 3 & 2 & 11 \\
IS9830 & 1 & 1 & 1 & 0 & 0 & 0 & 0 & 1 & 4 & 1 & 12 \\
AG-8 & 1 & 0 & 1 & 1 & 0 & 1 & 0 & 0 & 3 & 2 & 13 \\
N13 & 1 & 0 & 0 & 1 & 1 & 0 & 0 & 1 & 4 & 1 & 14 \\
Wad Ahmed & 1 & 0 & 1 & 1 & 0 & 0 & 0 & 0 & 4 & 2 & 15 \\
Tabat & 1 & 0 & 0 & 1 & 0 & 0 & 1 & 0 & 3 & 3 & 16 \\
\hline
\end{tabular}

Significance of the genotypic variance indicated that genotypes are different in their genetic potentials. The significance of genotype $x$ environment variance indicated that genotypes respond differently to the environments (Table 4). Genotype $x$ environment analysis confirmed that $\mathrm{T}_{1} \mathrm{BC}_{3} \mathrm{~S}_{4^{\prime}} \mathrm{AG}_{2} \mathrm{BC}_{3} \mathrm{~S}_{4^{\prime}}$
$\mathrm{AG} \mathrm{BC} \mathrm{S}_{3} \mathrm{~S}_{4}$ and $\mathrm{W}_{2} \mathrm{BC}_{3} \mathrm{~S}_{4^{\prime}}$ are not only superior in Striga prone areas but also have wide ranges of adaptation (Table 4).

\section{Adaptability and stability}

Multi-location testing and evaluation of varieties aimed 
Mohamed et al. Journal of Plant Science \& Molecular Breeding 2014,

Table 4. Mean squares from combined analysis of variance for grain yield in the nine environments.

\begin{tabular}{lllll}
\hline Sources of variation & df & MS & F Value & $\boldsymbol{P}>\mathbf{F}$ \\
\hline Environments & 8 & $9117730.499^{\star *}$ & 608.76 & 0.0001 \\
Replications & 18 & 50717.2999 & 3.39 & 0.0001 \\
Genotypes (G) & 15 & $1237178.981^{\star *}$ & 82.60 & 0.0001 \\
GxE & 120 & $118404.801^{\star *}$ & 7.91 & 0.0001 \\
\hline
\end{tabular}

at identifying genotypes that consistently produce stable yields over a range of diverse environments. [3] used a model where $b=$ regression coefficient is considered a parameter of the genotype response or adaptation. They also used the deviation from regression $\left(S^{2} d\right)$ to estimate stability. Regression coefficient was employed by [9] who used the variety and the magnitude of mean yield to identify adaptability and stability. They defined an ideal or an average stable variety as one with $b=1, S^{2} d=0$ and average mean is higher than the overall mean of grain yield of the trials.

In this study, generally the genotypes, $\mathrm{W}_{2} \mathrm{BC}_{3} \mathrm{~S}_{4^{\prime}}, \mathrm{T} 1 \mathrm{BC} \mathrm{C}_{3} \mathrm{~S}_{4^{\prime}}$ $\mathrm{AG} \mathrm{BC} \mathrm{S}_{3} \mathrm{~S}_{4^{\prime}} \mathrm{AG} 2 \mathrm{BC}_{3} \mathrm{~S}_{4^{\prime}}$, had b values of $1.41,1.23,1.35$, and 1.21, respectively, which are significantly greater than 1 , indicating that they are more responsive to environmental changes and are more adaptive (Table 5). However, results indicated clear differences in slopes of the regression lines between the tested lines and the checks. Striga resistant checks SRN39 and IS9830 had slopes of regression of 0.651 and 0.724 , respectively, which are less than unity indicating that they are more adapted to Striga prone areas, but produced below average mean grain yield and revealed instability (Table 5). Genotypes, $\mathrm{W}_{2} \mathrm{BC}_{3} \mathrm{~S}_{4^{\prime}}$ $\mathrm{T} 1 \mathrm{BC}_{3} \mathrm{~S}_{4^{\prime}} \mathrm{AG} \mathrm{BC} \mathrm{C}_{3} \mathrm{~S}_{4^{\prime}} \mathrm{AG}_{2} \mathrm{BC}_{3} \mathrm{~S}_{4}$ had $\mathrm{b}$ values greater than unity and deviations from regression far lower than those of the checks Tabat, Wad Ahmed and AG-8, and closer to $\mathrm{S}^{2} \mathrm{~d}=0$, and grain yield means of, $2817.9,2565.6,2463.3,2337.6 \mathrm{~kg} / \mathrm{h}$, which are above the overall mean of $4510.7 \mathrm{~kg} / \mathrm{ha}$ of the trials. Thus these genotypes have better responses in favorable and unfavorable environments and are therefore, adaptable and predictable (high $\mathrm{R}^{2}$ values) (Table 5). It is concluded that these sorghum lines would be preferred because of high yield potentials and wide range of adaptation.

\section{Striga count}

Analysis of variance of individual and combined environments revealed significant reduction in $10 \mathrm{BC}_{3} \mathrm{~S}_{4}$ lines, N13, SRN39 and IS9830 relative to susceptible checks, Wad-Ahmed, Tabat, AG-8 (Table 6). This indicates that these $10 \mathrm{BC}_{3} \mathrm{~S}_{4}$ lines are as

Table 5. Mean squares from combined analysis of variance for grain yield in the nine environments.

\begin{tabular}{llllllll}
\hline Genotype & mean & Slope & SE & MS-TXL & MS-REG & MS-DEV & R$^{2}(\%)$ \\
\hline T1BC $_{3} \mathrm{~S}_{4}$ & 1035 & $1.23^{*}$ & 0.137 & 35253.75 & 83505.30 & 28360.67 & 30 \\
AG1BC $_{3} \mathrm{~S}_{4}$ & 781 & 1.11 & 0.081 & 11186.74 & 19429.78 & 10009.17 & 22 \\
AG2BC $_{3} \mathrm{~S}_{4}$ & 982 & $1.21^{*}$ & 0.083 & 17115.01 & 64050.77 & 10409.90 & 47 \\
AG3BC $_{3} \mathrm{~S}_{4}$ & 935 & 0.938 & 0.269 & 96856.34 & 5848.55 & 109857.45 & 1 \\
W1BC $_{3} \mathrm{~S}_{4}$ & 789 & 0.940 & 0.078 & 8839.5 & 5383.01 & 9333.32 & 8 \\
AG4BC $_{3} \mathrm{~S}_{4}$ & 795 & 1.14 & 0.095 & 15690.11 & 28679.91 & 13834.43 & 23 \\
AG5BC $_{3} \mathrm{~S}_{4}$ & 880 & $1.10^{*}$ & 0.042 & 4233.43 & 15236.32 & 2661.58 & 45 \\
AG6BC $_{3} \mathrm{~S}_{4}$ & 1078 & $1.35^{*}$ & 0.118 & 41887.26 & 186241.80 & 21265.18 & 56 \\
T2BC $_{3} \mathrm{~S}_{4}$ & 784 & 0.955 & 0.065 & 5974.10 & 3019.16 & 6396.23 & 6 \\
W2BC $_{3} \mathrm{~S}_{4}$ & 1184 & $1.41^{*}$ & 0.133 & 56212.35 & 260643.42 & 27007.91 & 58 \\
SRN39 $_{\text {IS9830 }}$ & 596 & $0.651^{*}$ & 0.076 & 30842.15 & 184794.11 & 8849.01 & 75 \\
N13 & 693 & $0.724^{*}$ & 0.094 & 26135.27 & 115889.70 & 13313.20 & 55 \\
Tabat & 358 & 0.922 & 0.125 & 21838.20 & 9143.60 & 23651.71 & 5 \\
Wad Ahmed & 620 & 0.864 & 0.188 & 50327.16 & 27930.96 & 53526.62 & 7 \\
AG-8 & 600 & 0.839 & 0.217 & 67332.08 & 39593.26 & 71294.77 & 7 \\
Over all mean & 796 & -- & -- & -- & -- & -- & -- \\
\hline Slope-b slop & & & & & &
\end{tabular}

Slope-b slopes of regression of variety means on site index values

${ }^{*}$ Indicates slope significantly different from the slope for the overall regression which is 1.00

MS-TXL contribution of each variety to interaction

MS-REG contribution of each variety to the regression component of the treatment by location interaction

MS-DEV Deviations from regression component interaction

$\mathrm{R}^{2}$ Squared correlation between residual from the main effects model and its site index 
Mohamed et al. Journal of Plant Science \& Molecular Breeding 2014, http://www.hoajonline.com/journals/pdf/2050-2389-3-3.pdf

Table 6. Mean emerged Striga plants (plants $/ \mathrm{m}^{2}$ ), averaged over nine environments.

\begin{tabular}{|c|c|c|c|c|c|c|c|c|c|c|}
\hline ENT/OB & MED09 & MED010 & MED011 & SIN010 & SIN011 & DAM09 & DAM010 & DAM011 & GAD011 & COMB \\
\hline $\mathrm{T}_{1} \mathrm{BC}_{3} \mathrm{~S}_{4}$ & $2.00^{\mathrm{b}}$ & $2.67^{b}$ & $7.50^{\mathrm{b}}$ & $0.00^{\mathrm{b}}$ & $0.00^{\mathrm{c}}$ & $0.33^{\mathrm{b}}$ & $2.33^{\text {fgh }}$ & $2.00^{\mathrm{a}}$ & $44.00^{\mathrm{a}}$ & $9.30^{\mathrm{b}}$ \\
\hline $\mathrm{AG}_{1 B C} \mathrm{~S}_{4}$ & $3.00^{\mathrm{b}}$ & $3.33^{\mathrm{b}}$ & $11.33^{\mathrm{ab}}$ & $0.00^{\mathrm{b}}$ & $0.00^{c}$ & $1.67^{b}$ & $3.33^{\mathrm{def}}$ & $11.00^{\mathrm{a}}$ & $36.57^{a}$ & $8.69^{b}$ \\
\hline $\mathrm{AG} 2 \mathrm{BC}_{3} \mathrm{~S}_{4}$ & $2.00^{\mathrm{b}}$ & $3.00^{\mathrm{b}}$ & $11.00^{\mathrm{ab}}$ & $0.00^{\mathrm{b}}$ & $0.00^{c}$ & $0.67^{b}$ & $3.33^{\text {def }}$ & $21.33^{\mathrm{a}}$ & $12.30^{\mathrm{a}}$ & $6.85^{b}$ \\
\hline $\mathrm{AG} \mathrm{BC}_{3} \mathrm{~S}_{4}$ & $2.67^{b}$ & $2.67^{b}$ & $7.83^{\mathrm{b}}$ & $0.00^{\mathrm{b}}$ & $0.00^{c}$ & $0.67^{b}$ & $3.00^{\text {efg }}$ & $19.67^{a}$ & $25.80^{\mathrm{a}}$ & $7.50^{\mathrm{b}}$ \\
\hline $\mathrm{W} 1 \mathrm{BC}_{3} \mathrm{~S}_{4}$ & $3.00^{\mathrm{b}}$ & $3.33^{\mathrm{b}}$ & $11.00^{\mathrm{ab}}$ & $0.00^{\mathrm{b}}$ & $0.00^{c}$ & $0.00^{\mathrm{b}}$ & $4.67^{\mathrm{bcd}}$ & $23.67^{\mathrm{a}}$ & $35.67^{\mathrm{a}}$ & $9.89^{b}$ \\
\hline $\mathrm{AG}_{4} \mathrm{BC}_{3} \mathrm{~S}_{4}$ & $2.67^{b}$ & $3.33^{\mathrm{b}}$ & $11.17^{\mathrm{ab}}$ & $0.00^{\mathrm{b}}$ & $0.00^{c}$ & $0.67^{b}$ & $6.00^{\mathrm{b}}$ & $11.67^{a}$ & $41.70^{\mathrm{a}}$ & $9.45^{b}$ \\
\hline $\mathrm{AG} \mathrm{BC}_{3} \mathrm{~S}_{4}$ & $3.33^{\mathrm{b}}$ & $3.00^{\mathrm{b}}$ & $11.33^{\mathrm{ab}}$ & $0.00^{\mathrm{b}}$ & $0.00^{c}$ & $0.67^{b}$ & $3.67^{\text {cde }}$ & $17.67^{a}$ & $43.57^{\mathrm{a}}$ & $10.17^{b}$ \\
\hline $\mathrm{AG} \mathrm{BC}_{3} \mathrm{~S}_{4}$ & $2.00^{\mathrm{b}}$ & $2.67^{\mathrm{b}}$ & $7.167^{\mathrm{b}}$ & $0.00^{\mathrm{b}}$ & $0.00^{c}$ & $0.67^{b}$ & $3.33^{\text {def }}$ & $12.67^{\mathrm{a}}$ & $43.10^{\mathrm{a}}$ & $8.45^{\mathrm{b}}$ \\
\hline $\mathrm{T}_{2} \mathrm{BC}_{3} \mathrm{~S}_{4}$ & $3.00^{\mathrm{b}}$ & $3.33^{\mathrm{b}}$ & $11.83^{\mathrm{ab}}$ & $0.00^{\mathrm{b}}$ & $0.00^{c}$ & $1.00^{\mathrm{b}}$ & $1.33^{\mathrm{hi}}$ & $22.67^{\mathrm{a}}$ & $33.90^{\mathrm{a}}$ & $9.51^{b}$ \\
\hline $\mathrm{W} 2 \mathrm{BC}_{3} \mathrm{~S}_{4}$ & $2.33^{\mathrm{b}}$ & $2.33^{\mathrm{b}}$ & $7.33^{\mathrm{b}}$ & $0.00^{\mathrm{b}}$ & $0.00^{c}$ & $1.00^{\mathrm{b}}$ & $5.33^{\mathrm{bcd}}$ & $11.33^{\mathrm{a}}$ & $28.46^{\mathrm{a}}$ & $7.01^{b}$ \\
\hline SRN39 & $2.67^{\mathrm{b}}$ & $2.67^{\mathrm{b}}$ & $8.00^{\mathrm{b}}$ & $0.00^{\mathrm{b}}$ & $0.00^{c}$ & $1.33^{\mathrm{b}}$ & $1.00^{\mathrm{i}}$ & $11.00^{\mathrm{a}}$ & $25.87^{\mathrm{a}}$ & $6.43^{b}$ \\
\hline IS9830 & $3.00^{\mathrm{b}}$ & $3.33^{\mathrm{b}}$ & $7.17^{\mathrm{b}}$ & $0.00^{\mathrm{b}}$ & $0.00^{c}$ & $0.33^{b}$ & $2.00^{\mathrm{gh}}$ & $10.00^{\mathrm{a}}$ & $29.00^{\mathrm{a}}$ & $6.52^{b}$ \\
\hline N13 & $2.33^{\mathrm{b}}$ & $3.00^{\mathrm{b}}$ & $7.17^{\mathbf{b}}$ & $0.00^{\mathrm{b}}$ & $0.00^{c}$ & $1.33^{\mathrm{b}}$ & $4.33^{\mathrm{bcd}}$ & $9.33^{\mathrm{a}}$ & $24.90^{\mathrm{a}}$ & $6.29^{b}$ \\
\hline Tabat & $9.00^{\mathrm{a}}$ & $9.67^{\mathrm{a}}$ & $33.17^{\mathrm{ab}}$ & $4.67^{\mathrm{a}}$ & $10.33^{a}$ & $5.00^{\mathrm{a}}$ & $8.00^{\mathrm{a}}$ & $21.33^{\mathrm{a}}$ & $73.90^{\mathrm{a}}$ & $22.06^{\mathrm{a}}$ \\
\hline Wad Ahmed & $10.33^{\mathrm{a}}$ & $11.00^{\mathrm{a}}$ & $34.67^{\mathrm{a}}$ & $5.00^{\mathrm{a}}$ & $7.67^{\mathrm{ab}}$ & $5.00^{\mathrm{a}}$ & $5.67^{\mathrm{cb}}$ & $17.67^{\mathrm{a}}$ & $46.10^{\mathrm{a}}$ & $18.53^{\mathrm{a}}$ \\
\hline AG-8 & $9.67^{\mathrm{a}}$ & $9.0^{\mathrm{a}}$ & $30.33^{\mathrm{ab}}$ & $4.33^{\mathrm{a}}$ & $6.33^{\mathrm{b}}$ & $4.67^{\mathrm{a}}$ & $4.00^{\mathrm{bcd}}$ & $13.67^{a}$ & $57.43^{\mathrm{a}}$ & $17.86^{\mathrm{a}}$ \\
\hline $\mathrm{SE} \pm$ & 0.89 & 0.54 & 7.60 & 0.32 & 0.72 & 0.65 & 0.42 & 3.35 & 12.8 & 4.50 \\
\hline $\mathrm{CV}$ & 39.26 & 21.92 & 95.96 & 62.45 & 82.16 & 71.99 & 19.05 & 36.38 & 58.84 & 76.13 \\
\hline
\end{tabular}

** Means with different letters in the same column are significantly different at $P=0.05$

Striga resistant as the resistant checks,N13, SRN39 and IS9830. The lines $\left(\mathrm{BC}_{3} \mathrm{~S}_{4}\right)$ with only two major QTLs have the same level of resistance as the donor parent, and as the lines with four QTLs (including the 2 major QTLs). Targeting these 2 major QTLs will make map based cloning possible and ease inter and intra-specific gene transfer. However, it will give the trait a qualitative nature that could affect its durability.

\section{Agronomic traits}

Analysis of individual and combined experiments revealed significant differences $(P=0.05)$ among genotypes, environments and genotype $x$ environment for days to $50 \%$ flowering and plant height (Tables 7 and 8). Mean days to $50 \%$ flowering for each genotype at each environment and across environments is given in (Table 7). The selected genotypes, $\mathrm{WBC}_{3} \mathrm{~S}_{4^{\prime}}, \mathrm{T}_{1} \mathrm{BC}_{3} \mathrm{~S}_{4^{\prime}}$ and $\mathrm{AG} 2 \mathrm{BC}_{3} \mathrm{~S}_{4}$ have consistently earlier flowering time values than their recurrent parents, Wad Ahmed, Tabat and AG-8, across these Striga-infested environments (Table 7). The lines, $A G 2 \mathrm{BC}_{3} \mathrm{~S}_{4}$ and $\mathrm{AG} 6 \mathrm{BC} \mathrm{C}_{3}$ are significantly earlier than the Striga resistant checks SRN39 and IS9830, while $\mathrm{T}_{1 \mathrm{BC}} \mathrm{S}_{4}$ is significantly earlier than SRN39 and as early as IS9830. The line $\mathrm{W}_{2} \mathrm{BC}_{3} \mathrm{~S}_{4^{\prime}}$ is late maturing compared to Striga resistant checks SRN39 and IS9830. Comparing the genotypes with their recurrent parents, ranks starting with early are as follows; AG2BC $\mathrm{S}_{4}>\mathrm{AG}-8>\mathrm{AG}_{6 \mathrm{BC}} \mathrm{S}_{4}>\mathrm{T} 1 \mathrm{BC} \mathrm{C}_{3} \mathrm{~S}_{4}$ $>\mathrm{W}_{2} \mathrm{BC}_{3} \mathrm{~S}_{4}>$ Wad Ahmed AG2BC $\mathrm{S}_{4}>$ Tabat (Table 7).

\section{Verification trials}

Verification yield trials were initiated in season 2011/2012. These lines, $\mathrm{W}_{2} \mathrm{BC}_{3} \mathrm{~S}_{3^{\prime}}, \mathrm{T}_{1 \mathrm{BC}} \mathrm{S}_{3^{\prime}}, \mathrm{AG}_{2} \mathrm{BC}_{3} \mathrm{~S}_{3^{\prime}}$ and $\mathrm{AG} 6 \mathrm{BC} \mathrm{C}_{3} \mathrm{~S}_{3^{\prime}}$ were compared with Ajab-Sedo and Korokolo, at North Gedarif,
Tawawa, and South Gedarif, Doka (Table 1). Satisfactory grain yield was obtained even under unfavorable low inputs environments (Table 9). Farmers also reported that, $\mathrm{W}_{2} \mathrm{BC}_{3} \mathrm{~S}_{4^{\prime}}$ $\mathrm{T}_{1} \mathrm{BC}_{3} \mathrm{~S}_{4^{\prime}} \mathrm{AG}_{2} \mathrm{BC}_{3} \mathrm{~S}_{4^{\prime}}$ and $\mathrm{AG} 6 \mathrm{BC} \mathrm{S}_{3}$, have high kisra (sorghum bread) making qualities and plants are leafy with juicy and sweet stems which improve forage quality.

\section{Assessment of grain quality}

The physical characteristics of sorghum lines grains are presented in Table 10. Grain size or higher values of hectoliter weight (i.e., grain density or test weight) were 753.2, 740.0, 757.4, 734.4, and 747.6 g/L for lines, $\mathrm{T}_{1 B C_{3}} \mathrm{~S}_{4^{\prime}} \mathrm{AG} \mathrm{BC} \mathrm{C}_{3} \mathrm{~S}_{4^{\prime}}$ $\mathrm{AG} 6 \mathrm{BC}_{3} \mathrm{~S}_{4^{\prime}}, \mathrm{W}_{2} \mathrm{BC}_{3} \mathrm{~S}_{4^{\prime}}$ and $\mathrm{N} 13$, respectively. Generally, high test weight indicates sound, well-filled sorghum grain. The respective 1000-kernel weight for the lines was high and amounted to $25.78,37.00,37.28,27.08,27.36$, for $\mathrm{T}_{1 \mathrm{BC}} \mathrm{S}_{4^{\prime}}$ $\mathrm{AG} 2 \mathrm{BC}_{3} \mathrm{~S}_{4^{\prime}}, \mathrm{AG} \mathrm{BC}_{3} \mathrm{~S}_{4^{\prime}}, \mathrm{W}_{2} \mathrm{BC}_{3} \mathrm{~S}_{4^{\prime}}$ and N13, respectively (Table 10).

Grain hardness or texture as indicated by extraction rate for sorghum grain was $84.42 \%$ in N13 which was significantly harder than all introgression lines tested. $\mathrm{AG}_{6 \mathrm{BC}} \mathrm{S}_{4}$ with $81.65 \%$ extraction rate was significantly softer than the other three lines, $\mathrm{T}_{1 \mathrm{BC}} \mathrm{S}_{4^{\prime}} \mathrm{AG}^{\mathrm{B}} \mathrm{BC}_{3} \mathrm{~S}_{4^{\prime}}, \mathrm{W}_{2} \mathrm{BC}_{3} \mathrm{~S}_{4^{\prime}}$, where no significant differences were detected between them (Table 10).

\section{Chemical composition}

Chemical composition of sorghum grains, moisture, ash, and fat contents were normal in all the samples and were within the Sudanese standard recommended ranges. The ash content (2.39\%) was higher for $\mathrm{T}_{1} \mathrm{BC}_{3} \mathrm{~S}_{4}$ followed by 2.17, 1.94, 1.77, 1.64 and $1.57 \%$ for $A G 2 B C_{3} S_{4^{\prime}} A_{G 6 B C} S_{4^{\prime}} N 13$, and $\mathrm{W}_{2} \mathrm{BC}_{3} \mathrm{~S}_{4^{\prime}}$ respectively; no significant differences were found between 
Table 7. Mean days to $50 \%$ flowering of entries averaged over nine environments, Summary of level of superiority of genotypes for maturity across environments.

\begin{tabular}{|c|c|c|c|c|c|c|c|c|c|c|c|c|c|c|}
\hline ENT/OB & MED09 & \multicolumn{2}{|c|}{ MED010 } & \multicolumn{2}{|c|}{ MED011 } & SIN010 & SIN011 & \multicolumn{2}{|c|}{ DAM09 } & \multicolumn{2}{|c|}{ DAM010 } & DAM011 & GAD011 & COMB \\
\hline $\mathrm{T}_{1 B C} \mathrm{~S}_{4}$ & $53.0^{\mathrm{d}}$ & \multicolumn{2}{|c|}{$52.3^{\mathrm{d}}$} & \multicolumn{2}{|c|}{$57.3^{\mathrm{bc}}$} & $55.0^{\text {cd }}$ & $58.0^{\text {cde }}$ & \multicolumn{2}{|c|}{$47.0^{\mathrm{d}}$} & \multicolumn{2}{|c|}{$54.3^{\mathrm{de}}$} & $57.3^{\mathrm{ab}}$ & $64.0^{\mathrm{a}}$ & $55.4^{\mathrm{ef}}$ \\
\hline $\mathrm{AG}_{1 B C} \mathrm{~S}_{4}$ & $61.7^{\mathrm{c}}$ & \multicolumn{2}{|c|}{$61.7^{\mathrm{c}}$} & \multicolumn{2}{|c|}{$47.0^{\mathrm{e}}$} & $62.3^{\mathrm{b}}$ & $67.0^{\mathrm{a}}$ & \multicolumn{2}{|c|}{$70.0^{\mathrm{a}}$} & \multicolumn{2}{|c|}{$65.0^{\mathrm{ab}}$} & $47.0^{\mathrm{e}}$ & $63.0^{\mathrm{a}}$ & $60.3^{c}$ \\
\hline $\mathrm{AG}_{2} \mathrm{BC}_{3} \mathrm{~S}_{4}$ & $52.7^{\mathrm{d}}$ & \multicolumn{2}{|c|}{$52.6^{\mathrm{d}}$} & \multicolumn{2}{|c|}{$51.3^{\mathrm{de}}$} & $46.3^{\mathrm{f}}$ & $49.3^{\mathrm{g}}$ & \multicolumn{2}{|c|}{$49.3^{\text {cd }}$} & \multicolumn{2}{|c|}{$63.0^{\mathrm{abcd}}$} & $51.3^{\mathrm{de}}$ & $49.7^{\mathrm{a}}$ & $50.6^{\mathrm{i}}$ \\
\hline $\mathrm{AG}_{3} \mathrm{BC}_{3} \mathrm{~S}_{4}$ & $48.3^{\mathrm{d}}$ & \multicolumn{2}{|c|}{$53.0^{\mathrm{d}}$} & \multicolumn{2}{|c|}{$61.7^{\mathrm{ab}}$} & $52.0^{\mathrm{e}}$ & $53.0^{\mathrm{fg}}$ & \multicolumn{2}{|c|}{$48.3^{c}$} & \multicolumn{2}{|c|}{$50.3^{e}$} & $61.7^{\mathrm{ab}}$ & $52.7^{\mathrm{a}}$ & $54.4^{\mathrm{fg}}$ \\
\hline $\mathrm{W} 1 \mathrm{BC}_{3} \mathrm{~S}_{4}$ & $70.3^{\mathrm{a}}$ & \multicolumn{2}{|c|}{$68.3^{\mathrm{b}}$} & \multicolumn{2}{|c|}{$50.0^{\mathrm{de}}$} & $51.0^{\mathrm{e}}$ & $54.3^{\mathrm{efg}}$ & \multicolumn{2}{|c|}{$50.0^{\mathrm{cd}}$} & \multicolumn{2}{|c|}{$57.7^{\mathrm{bcd}}$} & $50.0^{\mathrm{de}}$ & $56.0^{\mathrm{a}}$ & $55.7^{\mathrm{ef}}$ \\
\hline $\mathrm{AG}_{4} \mathrm{BC}_{3} \mathrm{~S}_{4}$ & $50.0^{\mathrm{d}}$ & 50. & & 46.7 & & $61.7^{\mathrm{b}}$ & $65.0^{\mathrm{ab}}$ & 70. & & 51.3 & & $46.7^{\mathrm{e}}$ & $68.3^{\mathrm{a}}$ & $58.0^{\mathrm{d}}$ \\
\hline $\mathrm{AG} \mathrm{BC}_{3} \mathrm{~S}_{4}$ & $52.0^{\mathrm{d}}$ & 52. & & 48.3 & & $51.3^{\mathrm{e}}$ & $55.0^{\mathrm{def}}$ & 52. & & 63.7 & & $48.3^{\mathrm{de}}$ & $62.7^{\mathrm{a}}$ & $53.3^{\mathrm{gh}}$ \\
\hline $\mathrm{AG} \mathrm{BC}_{3} \mathrm{~S}_{4}$ & $50.3^{d}$ & 54. & & 53.7 & & $51.0^{\mathrm{e}}$ & $55.3^{\mathrm{def}}$ & 50. & & 58.3 & & $53.7^{\mathrm{dc}}$ & $49.3^{\mathrm{a}}$ & $52.3^{\mathrm{h}}$ \\
\hline $\mathrm{T}_{2} \mathrm{BC}_{3} \mathrm{~S}_{4}$ & $50.3^{\mathrm{d}}$ & $54.3^{3}$ & & 64.0 & & $55.0^{\text {cd }}$ & $59.7^{\mathrm{bcd}}$ & 50. & & 53.0 & & $64.0^{\mathrm{a}}$ & $56.0^{\mathrm{a}}$ & $56.6^{\mathrm{de}}$ \\
\hline $\mathrm{W} 2 \mathrm{BC}_{3} \mathrm{~S}_{4}$ & $62.3^{c}$ & 61.3 & & 60.3 & & $65.0^{\mathrm{a}}$ & $69.0^{\mathrm{a}}$ & 68. & & 55.3 & & $60.3^{\mathrm{ab}}$ & $63.7^{\mathrm{a}}$ & $63.7^{\mathrm{b}}$ \\
\hline SRN39 & $66.7^{\mathrm{b}}$ & $68 .($ & & 46.0 & & $55.3^{\mathrm{c}}$ & $58.3^{\text {cde }}$ & 64. & & 62.7 & & $46.0^{\mathrm{e}}$ & $65.3^{\mathrm{a}}$ & $59.7^{\mathrm{c}}$ \\
\hline IS9830 & $60.3^{c}$ & 66.7 & & 47.3 & & $56.0^{c}$ & $60.3^{\mathrm{bcd}}$ & 51. & & 49.3 & & $47.3^{\mathrm{e}}$ & $56.0^{\mathrm{a}}$ & $55.0^{\mathrm{ef}}$ \\
\hline N13 & $68.3^{\mathrm{b}}$ & 67.3 & & 64.3 & & $65.3^{\mathrm{a}}$ & $68.3^{\mathrm{a}}$ & 68. & & 67.7 & & $64.3^{\mathrm{a}}$ & $67.0^{\mathrm{a}}$ & $66.8^{\mathrm{a}}$ \\
\hline Tabat & $74.0^{\mathrm{a}}$ & 74.7 & & 66.0 & & $60.3^{\mathrm{b}}$ & $63.3^{\mathrm{abc}}$ & 69. & & 62.3 & & $66.0^{\mathrm{a}}$ & $72.0^{\mathrm{a}}$ & $67.2^{\mathrm{a}}$ \\
\hline Wad Ahmed & $70.0^{\mathrm{ab}}$ & $78 .($ & & 66.0 & & $60.7^{\mathrm{b}}$ & $63.3^{\mathrm{abc}}$ & 71. & & 63.7 & & $66.0^{\mathrm{a}}$ & $66.0^{\mathrm{a}}$ & $67.2^{\mathrm{a}}$ \\
\hline AG-8 & $50.7^{\mathrm{d}}$ & 52.7 & & 47.7 & & $52.3^{\mathrm{de}}$ & $55.3^{\mathrm{def}}$ & 51. & & 62.3 & & $47.7^{\mathrm{e}}$ & $56.7^{\mathrm{a}}$ & $53.0^{\mathrm{gh}}$ \\
\hline SE \pm & 1.04 & 1.2 & & 1.19 & & 0.62 & 1.17 & 0.7 & & 5.30 & & 1.19 & 4.24 & 1.63 \\
\hline $\mathrm{CV}$ & 3.07 & 3.4 & & 3.75 & & 1.91 & 3.37 & 2.2 & & 1.8 & & 3.75 & 12.43 & 4.85 \\
\hline Genotype & A & ab & Abc & B & bcd & $\mathrm{C}$ & cd & d & de & $\mathbf{E}$ & $\mathbf{F}$ & g & Ranking & \\
\hline $\mathrm{AG} \mathrm{BC}_{3} \mathrm{~S}_{4}$ & 1 & 0 & 0 & 0 & 0 & 2 & 0 & 2 & 2 & 1 & 1 & 0 & 2 & \\
\hline AG-8 & 1 & 0 & 0 & 0 & 0 & 2 & 0 & 2 & 1 & 2 & 1 & 0 & 3 & \\
\hline $\mathrm{AG} \mathrm{BC}_{3} \mathrm{~S}_{4}$ & 1 & 0 & 0 & 0 & 0 & 2 & 2 & 2 & 0 & 1 & 1 & 0 & 4 & \\
\hline $\mathrm{T}_{1} \mathrm{BC}_{3} \mathrm{~S}_{4}$ & 1 & 1 & 0 & 0 & 0 & 1 & 1 & 4 & 0 & 0 & 1 & 0 & 5 & \\
\hline $\mathrm{AG}_{3} \mathrm{BC}_{3} \mathrm{~S}_{4}$ & 1 & 2 & 0 & 0 & 0 & 1 & 1 & 2 & 0 & 1 & 0 & 1 & 6 & \\
\hline IS9830 & 1 & 0 & 0 & 0 & 2 & 4 & 0 & 0 & 0 & 2 & 0 & 0 & 7 & \\
\hline SRN39 & 1 & 0 & 0 & 4 & 0 & 1 & 0 & 0 & 0 & 2 & 1 & 0 & 8 & \\
\hline $\mathrm{W}_{1} \mathrm{BC}_{3} \mathrm{~S}_{4}$ & 2 & 0 & 0 & 1 & 0 & 0 & 2 & 0 & 1 & 1 & 1 & 1 & 9 & \\
\hline $\mathrm{T}_{2} \mathrm{BC}_{3} \mathrm{~S}_{4}$ & 3 & 0 & 0 & 0 & 1 & 0 & 3 & 2 & 0 & 0 & 0 & 0 & 10 & \\
\hline $\mathrm{AG}_{4} \mathrm{BC}_{3} \mathrm{~S}_{4}$ & 3 & 1 & 0 & 1 & 0 & 0 & 0 & 2 & 0 & 2 & 0 & 0 & 11 & \\
\hline $\mathrm{AG}_{1 B C} \mathrm{~S}_{4}$ & 4 & 0 & 0 & 1 & 0 & 2 & 0 & 0 & 0 & 2 & 0 & 0 & 12 & \\
\hline $\mathrm{W}_{2} \mathrm{BC}_{3} \mathrm{~S}_{4}$ & 5 & 2 & 0 & 0 & 0 & 2 & 0 & 0 & 0 & 0 & 0 & 0 & 13 & \\
\hline N13 & 7 & 0 & 0 & 2 & 0 & 0 & 0 & 0 & 0 & 0 & 0 & 0 & 14 & \\
\hline Wad Ahmed & 6 & 1 & 1 & 1 & 0 & 0 & 0 & 0 & 0 & 0 & 0 & 0 & 15 & \\
\hline Tabat & 7 & 0 & 1 & 1 & 0 & 0 & 0 & 0 & 0 & 0 & 0 & 0 & 16 & \\
\hline
\end{tabular}

${ }^{* *}$ Means with different letters in the same column are significantly different at $P=0.05$

the various ash contents at $P=0.05$ (Table 11).

Protein\%: highest protein content (14.24\%) was obtained in N13, which was significantly higher than all introgression lines tested, followed by lines, $A G 2 B C_{3} S_{4}(11.81 \%), W 2 B C_{3} S_{4}$ (11.79\%), $\mathrm{AG} \mathrm{BC}_{3} \mathrm{~S}_{4^{\prime}}(11.63 \%)$, and $\mathrm{T}_{1 \mathrm{BC}} \mathrm{S}_{4}(11.46 \%)$ (Table 11). It is evident that the last 4 lines are of good physical and chemical characteristics and with high nutritive values.

\section{Conclusion}

Climate change scenarios indicate that water shortage and short effective growing season will have predominant occurrence in sub-Saharan Africa, where Striga is endemic. This will definitely worsen Striga problem and necessitate the need for Striga resistant short-duration cereals such as sorghum. Sorghum lines $A G 6 B C_{3} S_{4^{\prime}}$ and $A G 2 B C_{3} S_{4}$ are promising varieties for drought prone areas of Sudan, because of their widely-effective Striga resistance and early maturity. Whereby sorghum lines $\mathrm{W} 2 \mathrm{BC} 3 \mathrm{~S} 3$ and $\mathrm{T} 1 \mathrm{BC} 3 \mathrm{~S} 3$, are promising varieties for Striga prone areas with intermediate to high rainfall and irrigated areas because of their wide Striga resistance and intermediate maturities. This coupled with their high yield potentials as well as their large white grains.

The use of DNA-based markers for the genetic analysis and manipulation of important agronomic traits has become an 
Mohamed et al. Journal of Plant Science \& Molecular Breeding 2014, http://www.hoajonline.com/journals/pdf/2050-2389-3-3.pdf

Table 8. Mean plant height $(\mathrm{cm})$ of entries averaged over nine environments.

\begin{tabular}{|c|c|c|c|c|c|c|c|c|c|c|}
\hline ENT/OB & MED09 & MED010 & MED011 & SIN010 & SIN011 & DAM09 & DAM010 & DAM011 & GAD2011 & COMB \\
\hline $\mathrm{T}_{1 B C} \mathrm{~S}_{4}$ & $131.00^{\mathrm{h}}$ & $133.33^{\mathrm{g}}$ & $132.66^{\mathrm{d}}$ & $152.66^{\mathrm{bcd}}$ & $155.67^{b c}$ & $152.66^{\mathrm{b}}$ & $146.00^{\text {bcd }}$ & $132.66^{\mathrm{d}}$ & $141.03^{\mathrm{abc}}$ & $141.71^{\mathrm{de}}$ \\
\hline $\mathrm{AG} 1 \mathrm{BC}_{3} \mathrm{~S}_{4}$ & $138.30^{\text {gh }}$ & $136.66^{\mathrm{g}}$ & $135.00^{\mathrm{d}}$ & $127.57^{\text {efg }}$ & $130.57^{\mathrm{cd}}$ & $91.66^{d}$ & $131.66^{\text {cde }}$ & $135.00^{\mathrm{d}}$ & $139.53^{\mathrm{abc}}$ & $132.37^{\mathrm{f}}$ \\
\hline $\mathrm{AG}_{2} \mathrm{BC}_{3} \mathrm{~S}_{4}$ & $131.67^{\mathrm{h}}$ & $135.00^{\mathrm{g}}$ & $135.00^{\mathrm{d}}$ & $157.66^{\mathrm{bcd}}$ & $160.33^{\mathrm{cb}}$ & $150.00^{\mathrm{b}}$ & $139.00^{\mathrm{bcd}}$ & $135.00^{\mathrm{d}}$ & $168.17^{\mathrm{abc}}$ & $144.39^{d}$ \\
\hline $\mathrm{AG}_{3} \mathrm{BC}_{3} \mathrm{~S}_{4}$ & $153.30^{\mathrm{e}}$ & $155.00^{\mathrm{e}}$ & $153.33^{c}$ & $169.00^{\mathrm{bc}}$ & $171.67^{\mathrm{b}}$ & $147.00^{\mathrm{b}}$ & $152.00^{\mathrm{bc}}$ & $153.33^{\mathrm{c}}$ & $177.73^{\mathrm{abc}}$ & $154.63^{\mathrm{c}}$ \\
\hline $\mathrm{W}_{1 \mathrm{BC}_{3} \mathrm{~S}_{4}}$ & $173.30^{c}$ & $175.00^{c}$ & $156.66^{c}$ & $144.66^{\mathrm{bcd}}$ & $147.33^{\mathrm{bcd}}$ & $146.33^{\mathrm{b}}$ & $140.00^{\mathrm{bcd}}$ & $156.66^{c}$ & $158.00^{\mathrm{abc}}$ & $150.85^{\mathrm{c}}$ \\
\hline $\mathrm{AG}_{4} \mathrm{BC}_{3} \mathrm{~S}_{4}$ & $153.33^{e}$ & $153.33^{\text {ef }}$ & $153.33^{c}$ & $130.90^{\text {def }}$ & $133.57^{\mathrm{cd}}$ & $120.33^{c}$ & $108.33^{\mathrm{g}}$ & $153.33^{c}$ & $101.80^{\text {ef }}$ & $137.22^{\text {ef }}$ \\
\hline $\mathrm{AG} \mathrm{BC}_{3} \mathrm{~S}_{4}$ & $156.67^{\mathrm{de}}$ & $166.67^{\mathrm{d}}$ & $153.33^{c}$ & $172.33^{\mathrm{b}}$ & $175.00^{\mathrm{b}}$ & $154.00^{\mathrm{b}}$ & $155.66^{\mathrm{ab}}$ & $153.33^{c}$ & $181.80^{\mathrm{abc}}$ & $161.83^{\mathrm{b}}$ \\
\hline $\mathrm{AG} \mathrm{BC}_{3} \mathrm{~S}_{4}$ & $133.00^{\mathrm{h}}$ & $135.00^{\mathrm{g}}$ & $136.66^{d}$ & $141.56^{\text {cde }}$ & $144.90^{\mathrm{bcd}}$ & $107.33^{\text {cd }}$ & $114.66^{\mathrm{fg}}$ & $136.66^{d}$ & $102.07^{\mathrm{ef}}$ & $131.99^{f}$ \\
\hline $\mathrm{T}_{2} \mathrm{BC}_{3} \mathrm{~S}_{4}$ & $165.00^{d}$ & $156.66^{\mathrm{e}}$ & $156.66^{c}$ & $172.00^{\mathrm{b}}$ & $175.00^{\mathrm{b}}$ & $153.33^{b}$ & $155.66^{\mathrm{ba}}$ & $156.67^{\mathrm{c}}$ & $201.23^{\mathrm{a}}$ & $161.10^{\mathrm{b}}$ \\
\hline $\mathrm{W} 2 \mathrm{BC}_{3} \mathrm{~S}_{4}$ & $148.33^{\text {ef }}$ & $146.66^{\mathrm{f}}$ & $151.66^{c}$ & $138.66^{\mathrm{def}}$ & $141.67^{\mathrm{bcd}}$ & $118.00^{\mathrm{c}}$ & $120.0^{\mathrm{efg}}$ & $151.66^{\mathrm{c}}$ & $116.50^{\text {cde }}$ & $135.32^{\mathrm{f}}$ \\
\hline SRN39 & $143.33^{\mathrm{fg}}$ & $135.00^{\mathrm{g}}$ & $135.00^{\mathrm{d}}$ & $115.66^{\mathrm{fg}}$ & $128.67^{\mathrm{cd}}$ & $110.66^{\mathrm{cd}}$ & $127.66^{\text {def }}$ & $135.00^{\mathrm{d}}$ & $129.80^{\mathrm{bcd}}$ & $132.61^{\mathrm{f}}$ \\
\hline IS9830 & & & & & $226.13^{\mathrm{a}}$ & & $175.66^{\mathrm{a}}$ & & & $207.81^{\mathrm{a}}$ \\
\hline N13 & $227.67^{\mathrm{a}}$ & $233.33^{\mathrm{a}}$ & $225.00^{\mathrm{a}}$ & $232.47^{\mathrm{a}}$ & $235.47^{a}$ & $158.33^{\mathrm{b}}$ & $159.6 b^{a}$ & $225.00^{\mathrm{a}}$ & $177.87^{\mathrm{abc}}$ & $206.05^{a}$ \\
\hline Tabat & $150.00^{\text {ef }}$ & $146.66^{\mathrm{f}}$ & $150.00^{c}$ & $112.33^{\mathrm{g}}$ & $115.33^{\mathrm{d}}$ & $111.33^{\mathrm{cd}}$ & $111.33^{\mathrm{fg}}$ & $150.00^{c}$ & $110.77^{\text {cde }}$ & $125.05^{\mathrm{g}}$ \\
\hline Wad Ahmed & $156.67^{\mathrm{de}}$ & $151.67^{\text {ef }}$ & $153.33^{c}$ & $139.23^{\mathrm{def}}$ & $141.90^{\mathrm{bcd}}$ & $116.67^{\mathrm{c}}$ & $114.33^{\mathrm{fg}}$ & $153.33^{c}$ & $142.87^{\mathrm{abc}}$ & $137.48^{\text {ef }}$ \\
\hline AG-8 & $131.67^{\mathrm{h}}$ & $130.00^{\mathrm{g}}$ & $127.66^{\mathrm{d}}$ & $126.46^{\mathrm{efg}}$ & $129.40^{\mathrm{cd}}$ & $105.33^{\mathrm{cd}}$ & $122.33^{\mathrm{efg}}$ & $127.66^{\mathrm{d}}$ & $90.60^{f}$ & $121.24^{\mathrm{g}}$ \\
\hline $\mathrm{SE} \pm$ & 1.97 & 1.55 & 1.97 & 5.9 & 6.96 & 4.9 & 4.31 & 1.97 & 13.61 & 6.27 \\
\hline $\mathrm{CV}$ & 2.17 & 1.71 & 2.21 & 6.65 & 7.66 & 6.33 & 5.3 & 2.21 & 16.11 & 7.28 \\
\hline
\end{tabular}

** Means with different letters in the same column are significantly different at $P=0.05$

Table 9. Verification yield trials 2011/2012, S.GAD (Doka) and N.GAD (Twawa), Yield (kg/ha).

\begin{tabular}{llll}
\hline Genotype & DOKA & Genotype & TWOWA \\
\hline T1BC $_{3} \mathrm{~S}_{5}$ & $997.22^{\mathrm{a}}$ & AG2BC $_{3} \mathrm{~S}_{5}$ & $583.89^{\mathrm{a}}$ \\
W2BC $_{3} \mathrm{~S}_{5}$ & $989.29^{\mathrm{a}}$ & AG6BC $_{3} \mathrm{~S}_{5}$ & $580.72^{\mathrm{a}}$ \\
Korokolo & $96.79^{\mathrm{b}}$ & Korokolo & $76.12 \mathrm{~b}$ \\
Ajab-Sedo & $77.75 \mathrm{~b}$ & Ajab-Sedo & 12.31 \\
$\mathrm{SE} \pm$ & 12.78 & $\mathrm{SE} \pm$ & 6.70 \\
$\mathrm{CV}$ & 9.73 & $\mathrm{CV}$ & 8.84 \\
\hline
\end{tabular}

** Means with different letters in the same column are significantly different at $P=0.05$

Table 10. Physical characteristics of sorghum lines.

\begin{tabular}{llll}
\hline Entry & $\begin{array}{l}\text { 1000 kernel } \\
\text { wt. (g) }\end{array}$ & $\begin{array}{l}\text { Hectoliter } \\
\text { wt. (g) }\end{array}$ & $\begin{array}{l}\text { Extraction } \\
\text { rate Hardness }\end{array}$ \\
\hline $\mathrm{T} 1 \mathrm{BC}_{3} \mathrm{~S}_{4}$ & 25.78 & 753.2 & 83.35 \\
$\mathrm{AG}_{4} \mathrm{BC}_{3} \mathrm{~S}_{4}$ & 37.00 & 740.0 & 83.02 \\
$\mathrm{AG}_{3} \mathrm{BC}_{3} \mathrm{~S}_{4}$ & 37.28 & 757.4 & 83.02 \\
$\mathrm{~W}_{2} \mathrm{BC}_{3} \mathrm{~S}_{4}$ & 27.08 & 734.4 & 81.65 \\
$\mathrm{~N} 13$ & 27.36 & 747.6 & 84.42 \\
$\mathrm{CV}$ & 0.39 & 1.50 & 0.21 \\
$\mathrm{SE} \pm$ & 0.74 & 0.12 & 0.30 \\
\hline
\end{tabular}

increasingly useful tool in modern plant breeding. The greatest potential of molecular markers is to improve precision and to accelerate selection gain of desirable genotypes of quantitative
Table 11. Chemical composition of the sorghum lines.

\begin{tabular}{llllll}
\hline Samples & $\begin{array}{l}\text { Moisture } \\
(\%)\end{array}$ & $\begin{array}{l}\text { Ash } \\
(\%)\end{array}$ & $\begin{array}{l}\text { Protein } \\
(\%)\end{array}$ & $\begin{array}{l}\text { Fat } \\
(\%)\end{array}$ & $\begin{array}{l}\text { CHO } \\
(\%)\end{array}$ \\
\hline $\mathrm{T}_{1 B C} \mathrm{~S}_{4}$ & 6.63 & 2.39 & 11.46 & 3.65 & 72.79 \\
$\mathrm{AG}_{2} \mathrm{BC}_{3} \mathrm{~S}_{4}$ & 6.25 & 2.17 & 11.81 & 3.32 & 76.41 \\
$\mathrm{AG} \mathrm{BC}_{3} \mathrm{~S}_{4}$ & 5.93 & 1.94 & 11.63 & 3.39 & 77.10 \\
$\mathrm{~W}_{2} \mathrm{BC}_{3} \mathrm{~S}_{4}$ & 6.36 & 1.64 & 11.79 & 3.29 & 76.88 \\
$\mathrm{~N} 13$ & 5.74 & 1.77 & 14.24 & 3.72 & 74.52 \\
$\mathrm{CV}$ & 0.046 & 0.03 & 0.23 & 0.06 & 0.24 \\
$\mathrm{SE} \pm$ & 0.43 & 0.98 & 1.04 & 1.07 & 0.18 \\
\hline
\end{tabular}

trait loci (QTLs) that condition complex important traits. Through marker-assisted selection (MAS), more rapid transfer of traits from donor parents to more elite locally-adapted crop cultivars (or hybrid parents) is possible. Whereby, backcrossing is often the chosen method to introduce a new trait into a breeding program, in particular when the trait of interest comes from a parent that has poor agronomic background.

Two major QTLs contributing from 14\%-94\% of the trait, the lines $\left(\mathrm{BC}_{3} \mathrm{~S}_{4}\right)$ with only two major QTLs have the same level of resistance as the donor parent. Targeting these 2 major QTLs will make map based cloning possible and ease inter and intra specific gene transfer. However, it will give the trait a qualitative nature that affects its durability.

\section{Competing interests}

The authors declare that they have no competing interests. 
Mohamed et al. Journal of Plant Science \& Molecular Breeding 2014,

\section{Authors' contributions}

\begin{tabular}{|l|c|c|c|c|c|c|c|c|}
\hline Authors' contributions & AM & RA & OE & ES & CM & CWM & AE & CTH \\
\hline Research concept and design & $\checkmark$ & $\checkmark$ & -- & -- & $\checkmark$ & $\checkmark$ & $\checkmark$ & $\checkmark$ \\
\hline Collection and/or assembly of data & $\checkmark$ & $\checkmark$ & $\checkmark$ & $\checkmark$ & $\checkmark$ & $\checkmark$ & -- & $\checkmark$ \\
\hline Data analysis and interpretation & $\checkmark$ & $\checkmark$ & $\checkmark$ & $\checkmark$ & -- & -- & -- & $\checkmark$ \\
\hline Writing the article & $\checkmark$ & -- & -- & -- & -- & -- & -- & -- \\
\hline Critical revision of the article & $\checkmark$ & $\checkmark$ & $\checkmark$ & $\checkmark$ & $\checkmark$ & $\checkmark$ & $\checkmark$ & $\checkmark$ \\
\hline Final approval of article & $\checkmark$ & $\checkmark$ & $\checkmark$ & $\checkmark$ & $\checkmark$ & $\checkmark$ & $\checkmark$ & $\checkmark$ \\
\hline Statistical analysis & $\checkmark$ & $\checkmark$ & $\checkmark$ & $\checkmark$ & $\checkmark$ & $\checkmark$ & $\checkmark$ & $\checkmark$ \\
\hline
\end{tabular}

\section{Acknowledgement}

Authors would like to thank, Agricultural Research

Corporation of Sudan (ARC), the Association for Strengthening Agricultural Research in East and Central Africa (ASARECA), the International Crops Research Institute for the SemiArid Tropics (ICRISAT), Biosciences for east and central Africa (BecA) and the University of Khartoum ( $U$ of $K$ ) for providing financial and technical assistance for this work.

\section{Publication history}

Editor: Pushpendra K. Gupta, Ch. Charan Singh University, India. EIC: Yong Pyo Lim, Chungnam National University, Korea. Received: 26-Feb-2014 Revised: 10-Jun-2014

Accepted: 20-Jun-2014 Published: 07-Jul-2014

\section{References}

1. AACC. Approved Methods of the American Association of Cereal Chemists, 8th ed. St. Paul, MN, USA. 1986.

2. AOAC. Official Methods of Analysis of the Association of Official Analytical Chemists, 15th ed. Arlington, VA, USA. 1990.

3. Eberhart $S A$ and Russel WA. Stability parameters for comparing crop varieties. Crop Science. 1966; 6:36-40. I Pdf

4. Ehiwe $A O$ and Reichert RD. Variability in dehulling quality of cowpea, pigeon pea and mung bean cultivars determined with the Tangential Abrasive Dehulling Device. Cereal Chem. 1987; 64:86-90. | Article

5. Ejeta G. Breeding for Striga resistance in sorghum: Exploitation of an intricate host-parasite biology. Crop Sci. 2007; 47:S-216 - S-227. I Article

6. Ejeta $\mathrm{G}$ and Knoll J. Marker-assisted selection in sorghum: R.K. Varshney and R. Tuberosa (eds.) Genomics Applica. in Crops. 2007; 2:187-205.

7. Ejeta $G$ and Butler LG. Host plant resistance to Striga. In R.F. Barnes et al. (eds.) Internat.Crop Sci. I. CSSA, Madison, WI. 1993; 561-569.

8. Ejeta G, Butler LG, Hess DE and Vogler RK. Genetics and breeding stratigies for Striga resistance in sorghum. In: K. Ransom, L.J. Musselman, A.D. Worsham and C. Parker (eds.). Proceedings of the $5^{\text {th }}$ Internat. Symp. on parasitic weeds, CIMMYT Nairobi. 1991; 539.

9. Finlay KW and Wilkinson $\mathrm{G}$. The analysis in adaptation of plant breeding programs. Aust. J. agric. Res. 1963; 14:724-54. I Pdf

10. Haussmann BI, Hess DE, Omanya GO, Folkertsma RT, Reddy BV, Kayentao $\mathrm{M}$, Welz HG and Geiger HH. Genomic regions influencing resistance to the parasitic weed Striga hermonthica in two recombinant inbred populations of sorghum. Theor Appl Genet. 2004; 109:1005-16. | Article I PubMed

11. Haussmann G, Hess E, Seetharama N, Welz G and Geiger H. Construction of a combined sorghum linkage map from two recombinant inbred populations using AFLP, SSR, RFLP, and RAPD markers, and comparison with other sorghum maps. Theor Appl Genet. 2002; 105:629-637. | Article | PubMed

12. Haussmann BI, Mahalakshmi V, Reddy BV, Seetharama N, Hash CT and Geiger HH. QTL mapping of stay-green in two sorghum recombinant inbred populations. Theor Appl Genet. 2002; 106:133-42. | Article | PubMed
13. Mace ES, Xia L, Jordan DR, Halloran K, Parh DK, Huttner E, Wenzl P and Kilian A. DArT markers: diversity analyses and mapping in Sorghum bicolor. BMC Genomics. 2008; 9:26. | Article | PubMed Abstract | PubMed Full Text

14. Mace ES, HK Buhariwalla and JH Crouch. A High-Throughput DNA Extraction Protocol for Tropical Molecular Breeding Programs, Plant Molecular Biology Reporter. 2003; 21:459. | Article

15. Maiti. R.K, K.V. Ramaiah, S.S.Bisen and V.L. Chidle. A comparative study of haustorial development of Striga Asiatica (L.) Kuntz on sorghum cultivars. Annals of Botany. 1984; 54:447-457.

16. Mohamed A.H, GamarY.A, Elhassan O.M, Elgada M.H and Assar A.H.A. Performance of selected sorghum genotypes under rain-fed areas of Sudan. Life Sci. Int. J. 2011; 5:2119-2127.

17. Mohamed A.H and Gamar Y.A. Climate change: Selection of sorghum genotype with wide adaptation, AG-17, for rain fed areas of Sudan. Int. J. A. R., 2011; 1:143-155. | Pdf

18. Mohamed A.H. Identification and characterization of genetic variants in sorghum for specific mechanisms of Striga resistance. PhD thesis. Purdue University, West Lafayette, IN. 2002. | Article

19. Ramu P, Deshpande SP, Senthilvel S, JayashreeB, Billot C, Deu M, Ananda $L$, Reddy and Hash CT. In-silico mapping of important genes and markers available in the public domain for efficient sorghum breeding. Molecul. Breed. 2010; 26:409-418. | Article

20. Ahmed RAM. Fine mapping of quantitative trait loci (QTLs) associated with Striga resistance using molecular markers and introgression of resistance genes into sorghum (Sorghum bicolor (L.) Moench) in Sudan, PhD thesis, University of khartoum. 2013.

21. Press MC, Scholes JD and Riches CR. Current status and future prospects for management of parasitic weeds (Striga and Orobanche). In The world's worst weeds. Proceedings of an international Symposium, Hilton Brighton Metropole Hotel, UK, 12 November 2001; 71-88. | Article

\section{Citation:}

Mohamed A, Ali R, Elhassan O, Suliman E, Mugoya C, Masiga CW, Elhusien A and Hash CT. First products of DNA marker-assisted selection in sorghum released for cultivation by farmers in sub-saharan Africa. $J$ Plant Sci Mol Breed. 2014; 3:3. http://dx.doi.org/10.7243/2050-2389-3-3 